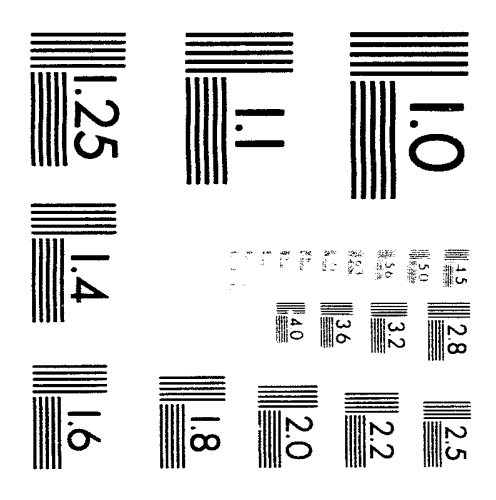



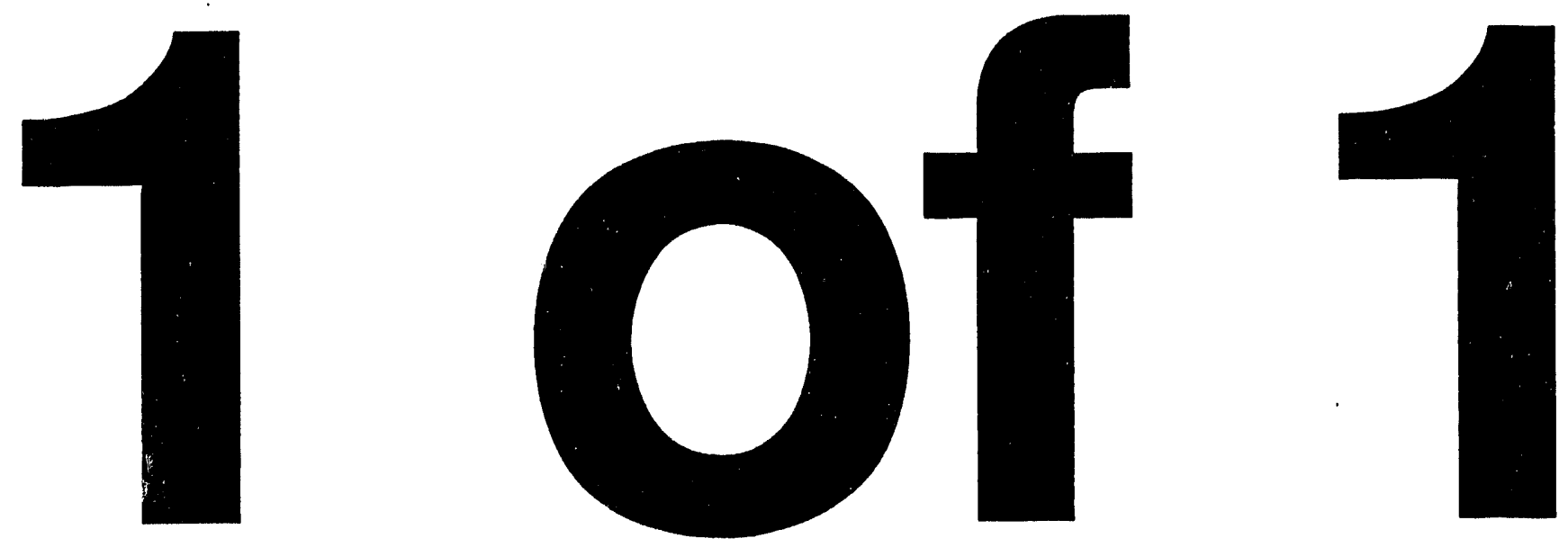
The Use of Computer Vision and Force Sensing for Tight Tolerance Assembly

RECE:VED
NOV 191993
OSTI

\author{
Jessica D. Bayliss
}

May 19, 1993

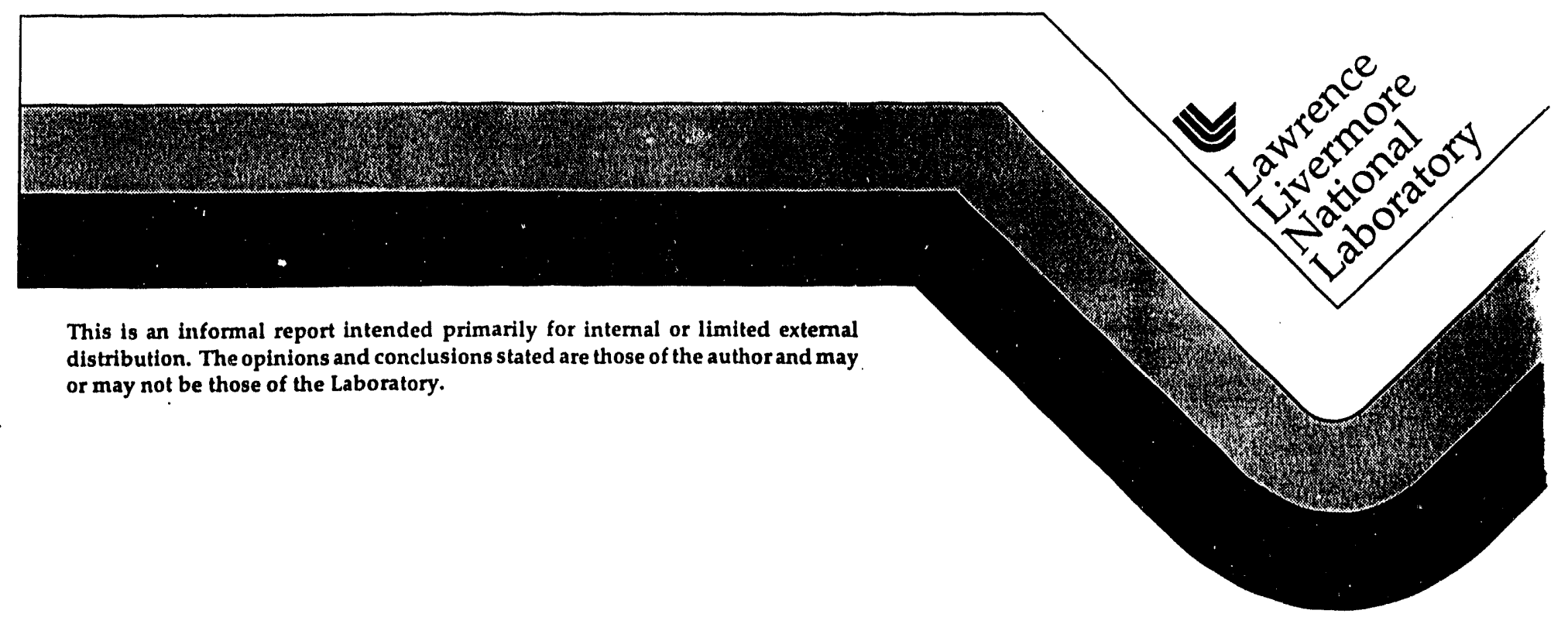




\section{DISCLAIMER}

This document was prepared as an account of work sponsored by an agency of the United States Government. Neither the United States Government nor the University of California nor any of their employees, makes any warranty, express or implied, or assumes any legal liability or responsibility for the accuracy, completeness, or usefulness of an y information, apparatus, product, or process disclosed, or represents that its use would not infringe privately owned rights. Reference herein to any specific commercial products, process, or service by trade name, trademark, manufacturer, or otherwise, does not necessarily constitute or imply it, endorsement, recommendation, or favoring by the United States Government or the University of California. The views and opinions of authors expressed herein do not necessarily state or reflect those of the United States Government or the University of California, and shall not be used for advertising or product endorsement purposes.

This report has been reproduced

directly from the best available copy.

Available to DOE and DOE contractors from the Office of Scientific and Technical Information

P.O. Box 62, Oak Ridge, TN 37831

Prices a vailable from (615) 576.8401, FTS 626.8401

Available to the public from the

National Technical Information Service

US. Department of Commerce

5285 Port Royal Rd.,

Springfield, VA 22161 


\title{
The Use of Computer Vision and Force Sensing for Tight Tolerance Assembly
}

\author{
Jessica D. Bayliss
}

\author{
California State University, Fresno \\ Lawrence Livermore National Laboratory \\ Livermore, California 94550
}

May 19, 1993

Prepared in partial fulfillment of the requirements of the Science and Engineering Research Semester under the direction of Shin-yee Lu, Research Mentor, at the Lawrence Livermore National Laboratory.

* This research was supported in part by an appointment to the U.S. Department of Energy Science and Engineering Research Semester (hereinafter called SERS) program administered by LLNL under Contract W-7405-Eng-48 with Lawrence Livermore National Laboratory.

By acceptance of this article, the publisher or recipient acknowledges the U.S. Goverment's right to retain a non-exclusive, royalty-free license in and to any copyright covering this article. 


\begin{abstract}
Computer vision and force control provide feedback for robot manipulation during the assembly of objects. Both techniques have weaknesses, but their complementary strengths enable them to work well together, achieving assembly with tight tolerances. For instance, camera resolution limits the accuracy of computer vision, but it can locate approximately where the part should be placed and is an excellent choice for coarse placement of the part. Force control senses the force induced by object contact and if used extensively could damage a delicate part, but when used for fine placement of an object, it compensates for the error in coarse placement. It is our goal to utilize the best features of force sensing and computer vision to reduce the error in placement of an object.

The results of placing a peg in a $0.15 \mathrm{~mm}$ tolerance hole with different camera resolutions will be presented. We have chosen to use computer vision to move the peg as close to its correct placement point as possible and force control to make minor adjustments, achieving the correct positioning of the peg.
\end{abstract}




\section{Background}

The peg-in-the-hole experiment demonstrates different methods for tight tolerance assembly. Each method solves a particular problem with tight tolerance assembly. For instance, Remote Center Compliance (RCC), or passive force sensing, uses a set of springs above a robot's gripper for tolerance to assembly error caused by slight angular or lateral misplacement of the peg as discussed in [8]. Active force sensing tries to solve the same problems while providing feedback information to perform more complex tasks in assembly as in [7]. In order to give active force sensing the same kind of error tolerance as RCC, a compliance/damping matrix has been proposed to provide the same kind of error tolerance in [6]. While these methods provide invaluable aid in inserting the peg once it is very close to the hole, they fail to offer any help in getting the peg close to the hole except to imply that a tight tolerance in robot performance and repeatability in placing the hole in the same location is necessary.

As an alternative to force sensing, computer vision could be used to place the peg in the hole. Here there are also problems, as computer vision is only as accurate as the image resolution being used. While a tight tolerance on robot accuracy can be exchanged for image accuracy in this case, the system will only work in a small amount of situations. For example, given the categories of possible errors that can be made in peg placement as shown in Figure 1, computer vision can only solve A, B, and C and then only if the angle of the peg can be determined correctly.

The main goal of this project is to develop a system for tight tolerance assembly that solves all the errors in Figure 1 without specifying ultra-tight tolerances on the equipment involved in placing and inserting the peg. The complementary use of force sensing and computer vision satisfy this goal. Computer vision can be used as the "eycs" of the robot for coarse placement of the peg and force control for fine placement and insertion of the peg. This way, the strengths of both methods can be used to provide a fault tolerant assembly system. 


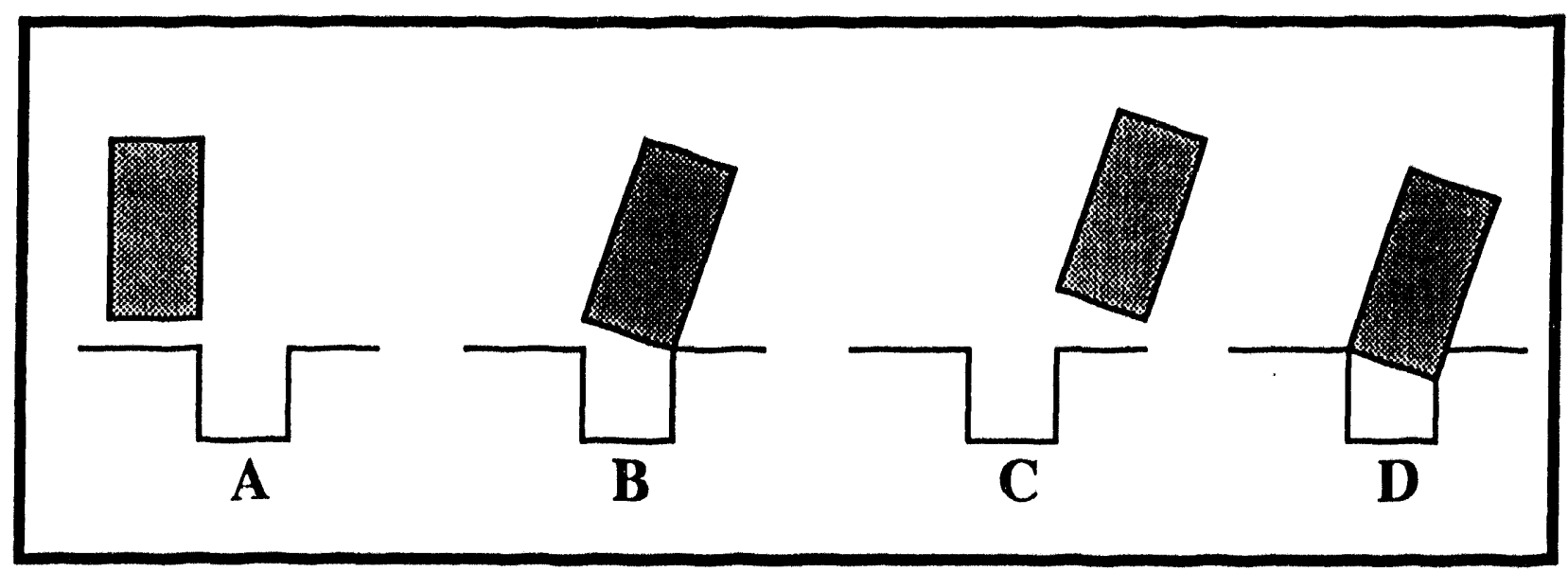

Figure 1 - A) The peg misaligned laterally with the hole, B) The peg angularly misaligned with the hole, C) The peg misaligned angularly and laterally with the hole, and D) The peg jammed in the hole because of angular misalignment. 


\section{General Approach}

In our experiment, a Zebra Zeroforce robotic arm, shown in Figure 2, is utilized for placement and insertion of an aluminum peg into a $0.15 \mathrm{~mm}$ tolerance hole in an aluminum box. Two cameras are set up to monitor the $x$ and $y$ directions of robot movement as shown in Figure 3 . The $\mathrm{z}$ axis is assumed to be $\mathrm{a}$ constant for the sake of simplicity and the $\mathrm{x}$ and $\mathrm{y}$ cameras are calibrated individually to make sure the peg will be in the camera's field of view when the robot moves it from its resting position. Before the robot moves, an image is taken and the box hole located. The peg is then brought to where the robot thinks the hole should be. To compensate for gear slippage and robot inaccuracy, a vision feedback loop makes sure the centroid of the peg is within two pixels of the centroid of the hole. Once the peg is over the hole, force control (both active and compliant force sensing) is used to insert the peg. As an added benefit, if the robot somehow manages to miss the hole, the peg is brought back up and computer vision again attempts to correctly place the peg over the hole.

\section{Computer Vision}

The computer vision system consists of two Sony XC-999 cameras with $6 \mathrm{~mm}$ lenses connected to Data Translation's DT2867 frame grabber board located in an expansion slot on a $486 \mathrm{pc}$. Image resolution is $640 \mathrm{X} 480$ pixels. The only component of the computer vision system that is not off-the-shelf is the vision processing software. The program used was an in-house program called Vision that is run in a Lisp environment from a Sparcstation-2 over a network. This was done for convenience as the necessary image processing techniques were already in existence in Vision. 


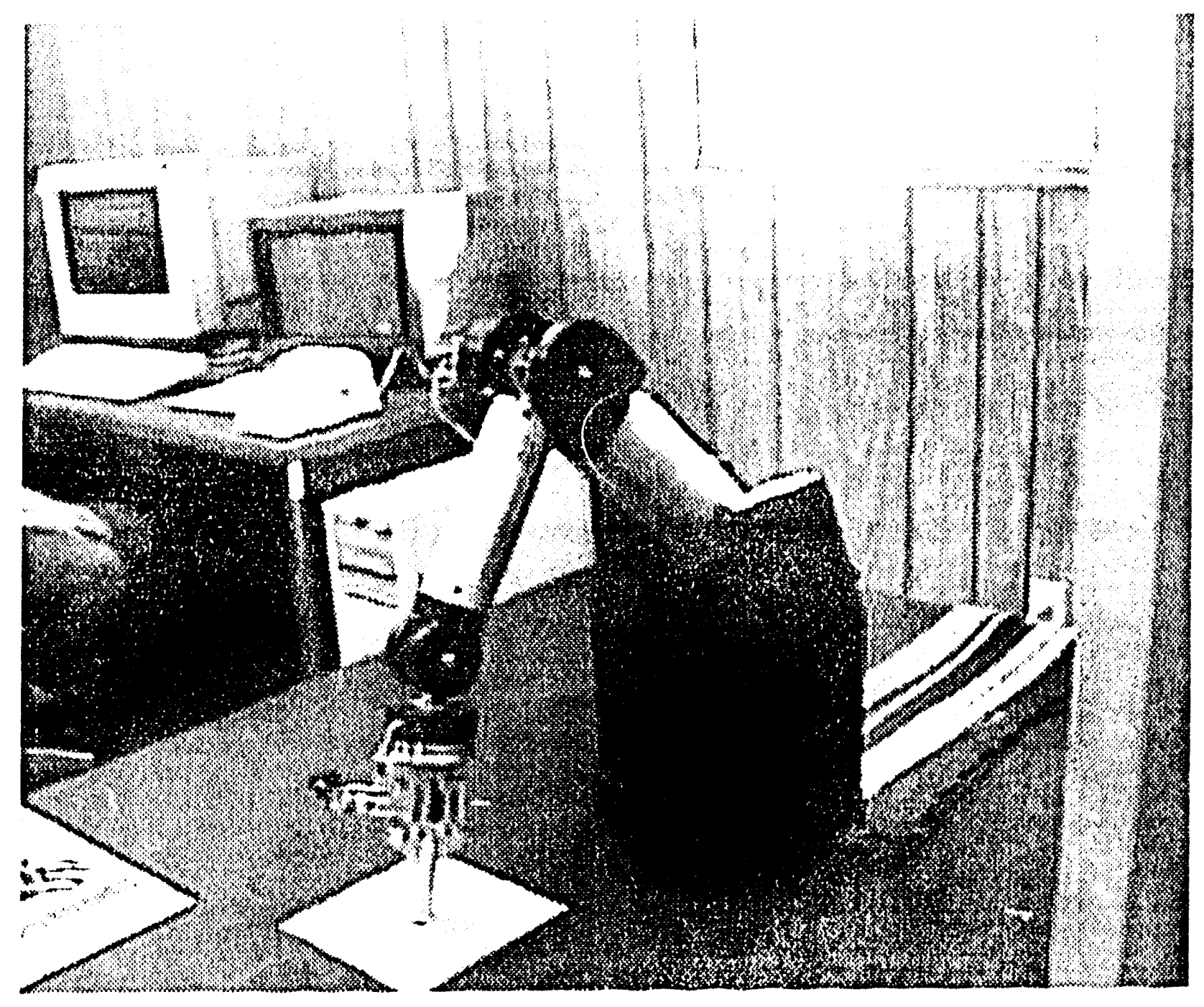

Figure 2 - zobra Zeroforce Robotic Arm loserting He Peg in the bos hole. 


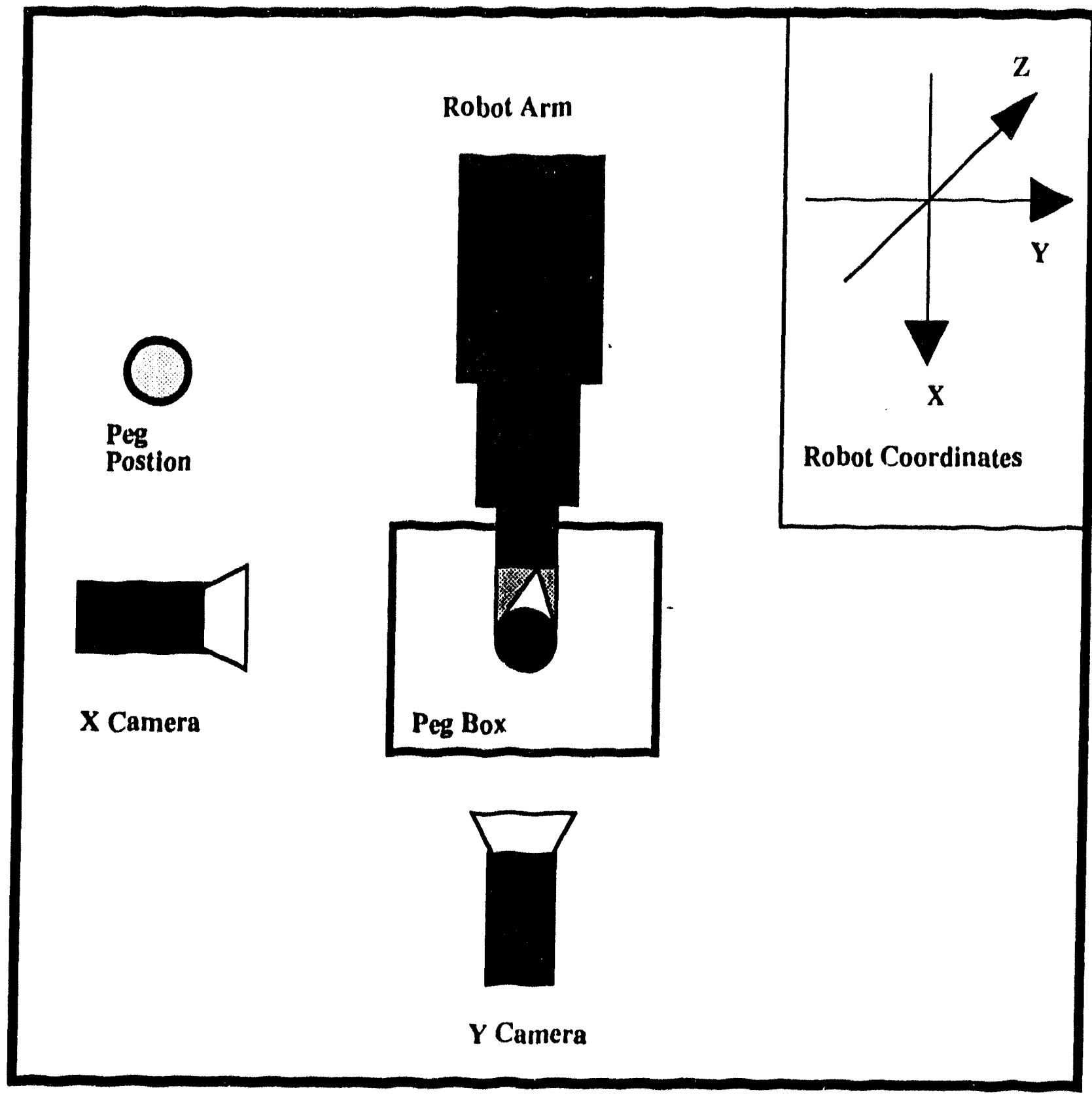

Figure 3 - Experimental Setup 


\section{Camera Calibration}

The computer vision feedback loop makes camera calibration almost unnecessary. However, camera calibration was found to improve the first time performance of placing the peg. Thus, both the $x$ and $y$ camera were calibrated independently with three calibration points. The location of a light source held in the robot's gripper was found in camera coordinates as well as robot coordinates. As we were concerned with a relatively small work area (the area of the $19.5 \mathrm{~mm}$ hole), the transformation from camera to robot coordinates was assumed to be linear and follow the equation of a line $(y=m x+b)$ where $y$ was an $x$ or $y$ robot coordinate, $m$ was the change in robot over the change in camera coordinates, $x$ was the camera coordinate, and $b$ was the constant offset gotten from looking at a particular point. Each camera was only responsible for one robot coordinate and the $z$ axis was assumed to be constant. We realize that there are more complex calibration techniques and camera setups as described in [2] and [5], but they did not seem necessary and would have reduced the flexibility of the system.

\section{Image Processing}

A $640 \times 480$ pixel image is taken of the box with the hole before the robot moves. Region-based methods, where the image is thresheld at a set value and segmented with an 8 neighbor pixel growing algorithm are used to find the regions of interest in the image. The hole is then located by filtering out non-circular regions that are outside of a 10 to 250 pixel region size and the centroid of the hole region is found. For a more in-depth description of region-based algorithms see [2] and [3]. This process is repeated for both the $x$ and $y$ cameras. The robot was then given the calculated $x$ and $y$ coordinates from the $x$ and $y$ cameras (respectively) and told to move the peg to that location.

Experimental testing showed that the hole algorithm was sturdy and could be used in a room with florescent lights and open windows with the cameras being from $90-450 \mathrm{~mm}(0.19 \mathrm{~mm} / \mathrm{pixel}$ 
to $0.83 \mathrm{~mm} / \mathrm{pixel}$ ) away from the hole. Changes in light are currently compensated for by changing the static threshold value of a camera, but in the future we hope to automate this process.

After locating the hole, the robot places the peg $1 \mathrm{~mm}$ over where the computer vision system claims the hole is in the $\mathrm{x}$ and $\mathrm{y}$ directions. A peg-finding algorithm uses a similar region-based method to the hole-finding algorithm except in the peg algorithm the background noise is first screened out by subtracting the image of the hole from the image of the peg. The centroid of the peg is found and compared with the location of the centroid of the hole. The peg image and processing steps for the $Y$ camera are shown in Figure 4. Region-based processing was used to locate the peg.

The peg algorithm is more sensitive to light than the hole algorithm and had a tendency to break down when the camera was greater than $450 \mathrm{~mm}$ away from the hole due to the wider field of view of the cameras. Surprisingly enough, even when the whole peg was not located due to shadows and reflections off of its aluminum surface, the centroid of the peg corresponded fairly close to that of the true centroid of the peg.

Computer vision feedback is used in case the robot's gears slip and it places the peg erroneously and because of slight error's in the transformation of camera to robot coordinates. An attempt to insert the peg is made when the centroid of the peg and the centroid of the hole are aligned within two pixels of each other. Two pixels was chosen because the image pixels could be off one pixel in any direction when taking the same picture and because a one pixel tolerance substantially raised the time for placement of the peg without any noticeable improvements in performance. Even with the two pixel tolerance, it took an average of four times to place the peg correctly over the hole. 


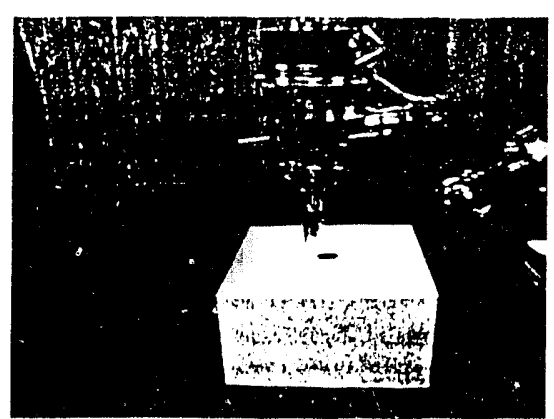

A

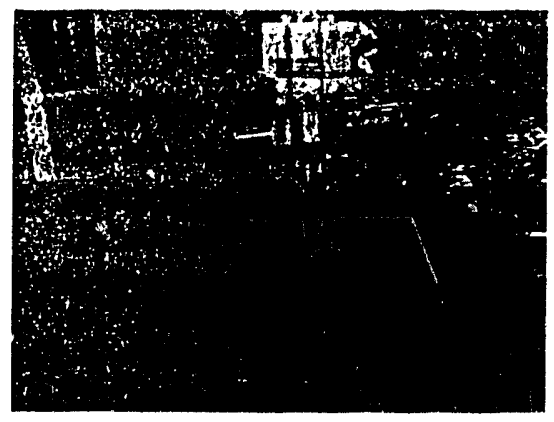

C

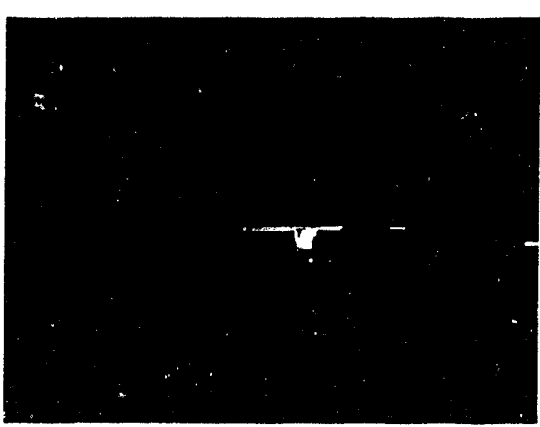

$\mathbf{E}$

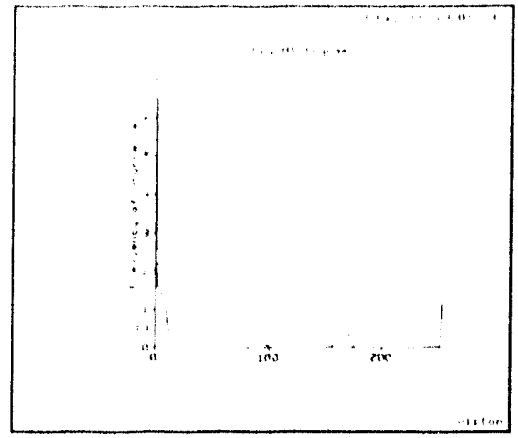

B
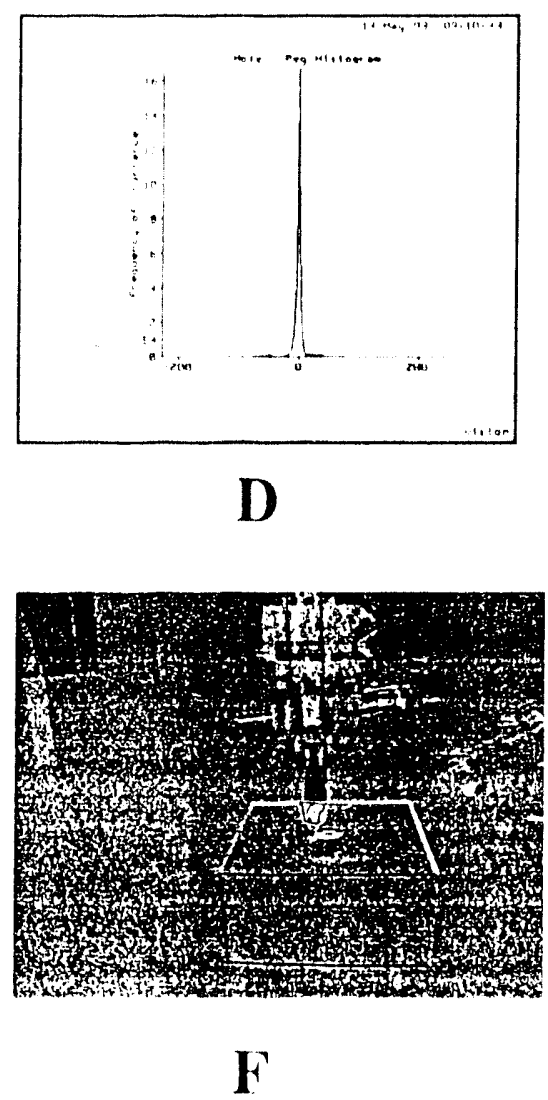

Figure 4 - A) The original peg image from the $Y$ camera, B) The histogram of image intensities for $A, C$ ) The difference of the peg and box images, I) The histogram of image intensities for B, E) The segmented regions for $\mathrm{C}$, and $\mathrm{F}$ ) The peg has been found. 


\section{Force Control}

The Zebra Zeroforce robot force sensor is mounted between the wrist and end-effector of the robotic arm and measures forces and moments in the $x, y$, and $z$ directions. To add a measure of compliant force sensing to the robot in the $\mathrm{x}$ and $\mathrm{y}$ directions, a rubber band was foam-taped to the inner part of the robot's grippers. Thus, both active and passive (compliant) force sensing were used to insert the peg into the hole.

The active force sensing was programmed in software on the $486 \mathrm{pc}$ to be compliant in the $\mathrm{x}$ and y positions while remaining stiff in the $z$ direction. This method of active force sensing with the compliant force sensing gives the error tolerance of Remote Centre Compliance without losing the sensory feedback that enables the robot arm to perform more complicated tasks. As a last measure protection against the peg jamming into the hole, a one degree wobble was added near the beginning of the insertion. In this way the error of Figure $1 \mathrm{~d}$ was solved.

Experimentally, the peg was inserted into the hole thirty-eight times with the cameras from 90$450 \mathrm{~mm}$ away and the peg never failed to enter the hole although it jammed at $450 \mathrm{~mm}$ when the true centroid of the peg was incorrect.

\section{Discussion}

The complementary use of computer vision and force sensing not only worked well in placing a peg into a tight tolerance hole, but provided for a fault tolerant and easily adaptable system. Surprisingly, this complementary usage has not been tried by many and when it is, usually computer vision dominates the experiment $[1,4]$. Although the peg-finding routine was somewhat sensitive to lighting conditions, it is possible to automate finding the peg threshold and make the algorithm applicable to a broader range of conditions.

Another area of concern is that of time. In this experiment it was near impossible to measure 
the time of an operation as the robot and frame grabber were located in a pc and the computer vision software was located on a Sun Sparcstation-2. The two were constantly communicating over a network which made timing the system very difficult. In the future, all software and hardware will be located on the same computer to enable real time processing of information.

\section{Acknowledgments}

This work is performed under the auspices of the U.S. Department of Energy and Engineering Research Semester (SERS) program administered by the Lawrence Livermore National Laboratory under Contract W-7405-Eng-48. The author would like to thank Shin-yee Lu, Robert Johnson, Jose Hernandez, and Eric Breitfeller for technical explanations and support.. 


\section{References}

1. R. L. Anderson, "Dynamic Sensing in a Ping-Pong Playing Robot", IEEE Transactions on Robotics and Automation.Vol. 5, No. 6, pp. 728-739, Dec. 1989.

2. D. H. Ballard and C. M. Brown, Computer Vision. Prentice-Hall, 1982.

3. P. J. Besl and R. c. Jain. "Range Image Segmentation", Machine Vision: Algorithms, Architectures, and Systems. Academic Press, pp. 221-257, 1988.

4. G. C. Burdea and H. J. Wolfson, "Solving Jigsaw Puzzles by a Robot", IEEE Transactions on Robotics and Automation.Vol. 5, No. 6, pp. 752-761, Dec. 1989.

5. B. Jahne, Digital Image Processing: Concepts, Algorithms, and Scientific Applications. New York: Springer-Verlag, pp. 23-39, 1991.

6. M. A. Peshkin, "Programmed Compliance for Error Corrective Assembly", IEEE Transactions on Robotics and Automation.Vol. 6, No. 4, pp. 473-482, Aug. 1990.

7. H. Van Brussel and J. Simons, "Automatic Assembly by Active Force Feedback Accommodation", Robot Sensors (International Trends in Manufacturing Technologies): Vol. 2 Tactile and Non-Vision, UK: IFSPublications, pp. 53-66, 1986.

8. D. E. Whitney and J. L. Nevins, "What is Remote Centre Compliance (RCC) and what Can It Do?", Robot Sensors (International Trends in Manufacturing Technologies): Vol. 2 Tactile and Non-Vision, UK: IFSPublications, pp. 3-15, 1986. 

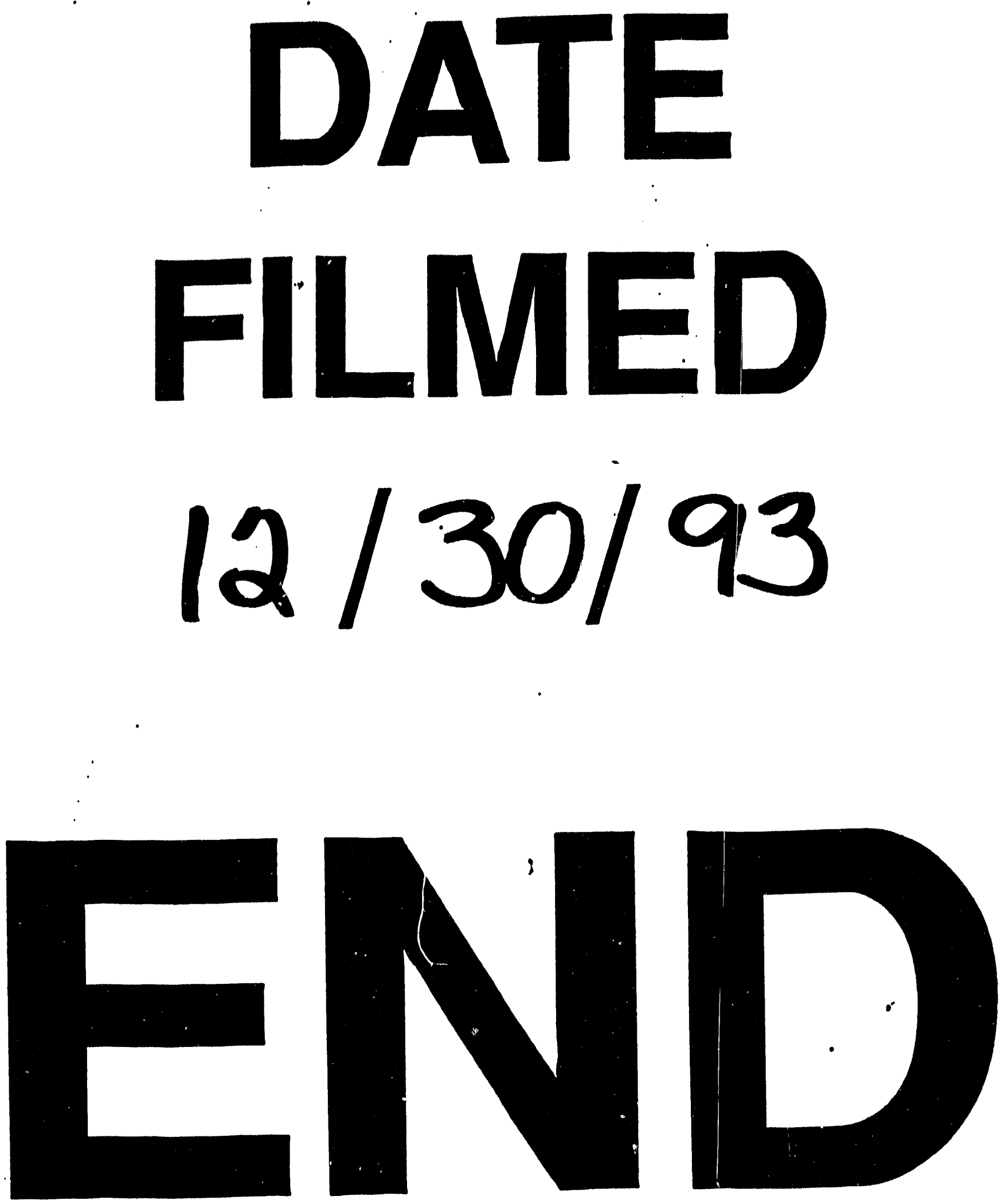
